\title{
Preparedness and Response to COVID-19 in Saudi Arabia: Lessons Learned from MERS- CoV
}

Abdullah A. Algaissi ${ }^{1,2}$, Naif Khalaf Alharbi ${ }^{3,4}$, Mazen Hassanain ${ }^{5}$, Anwar M. Hashem ${ }^{1,6^{*}}$

1 Vaccines and Immunotherapy Unit, King Fahd Medical Research Center, King Abdulaziz University, Jeddah, Saudi Arabia

2 Department of Medical Laboratories Technology, College of Applied Medical Sciences, Jazan University, Jazan, Saudi Arabia

3 Department of Infectious Disease Research, King Abdullah International Medical Research Center, Riyadh, Saudi Arabia

4 King Saud bin Abdulaziz University for Health Sciences, Riyadh, Saudi Arabia

5 Department of Surgery, Faculty of Medicine, King Saud University, Riyadh, Saudi Arabia

6 Department of Medical Microbiology and Parasitology, Faculty of Medicine, King Abdulaziz University, Jeddah, Saudi Arabia.

* Corresponding author: amhashem@kau.edu.sa

Key words: Saudi Arabia; COVID-19; MERS-CoV; control measures; travel restrictions

\section{Funding}

This work was funded by King Abdulaziz City for Science and Technology (KACST) grant number 09-1, which is a part of the Targeted Research Program (TRP).

Conflict of interest

None 


\begin{abstract}
Nearly three months have passed since the emergence of the severe acute respiratory syndromecoronavirus-2 (SARS-CoV-2), which caused the rapidly spreading Coronavirus Disease 2019 (COVID-19) pandemic. To date, there have been more than 550,000 confirmed cases and more than 25,000 deaths globally caused by COVID-19. Chinese health authorities, where the virus emerged, have taken prompt strict public health measures to control and prevent the spread of the outbreak. In the kingdom of Saudi Arabia, unprecedented precautionary strict measures were applied to slow virus entry and to mitigate the risk of the outbreak. Here, we review the experience learned during the Middle East Respiratory Syndrome Coronavirus (MERS-CoV) epidemic in Saudi Arabia, which has been in the country since 2012, and is expected to have helped the country to be well prepared for the current COVID-19 pandemic. We also discuss the country readiness, improvement in research and development, and the unprecedented rapid precautionary measures that have been taken by the Saudi government thus far.
\end{abstract}




\section{Emergence and global spread of coronaviruses}

Coronaviruses (CoVs) tend to emerge as an important human and animal pathogens, representing serious public health and economic concerns. Generally, CoVs cause gastrointestinal and respiratory infections with some infecting the central nervous system [1]. According to the latest viral taxonomy by the International Committee on the Taxonomy of Viruses (ICTV), CoVs are classified into four genera; alphacoronaviruses, betacoronaviruses, gammacoronaviruses and deltacoronaviruses based on the differences in phylogenetic relationships and genomic structures; and betacoronavirus contains 4 lineages (A, B, C and D) [2].

The first discovered coronavirus was the infectious bronchitis virus (IBV) in the 1930s as the primary cause of respiratory illnesses in domestic chickens [3]. Several other coronaviruses have been isolated and recognized as causative agents of respiratory or enteric infections in a variety of animals such as the porcine epidemic diarrhea virus (PEDV), the transmissible gastroenteritis virus (TGEV) and the bovine coronavirus (BCoV) [4-7]. In humans, coronaviruses mainly affect the respiratory tract causing diseases ranging from the mild common cold to fatal pneumonia $[8,9]$. The first human coronaviruses (hCoVs); hCoV-OC43 and hCoV-229E; were discovered in the 1960s as causative pathogens of the common cold [10,11]. In 2002-2003, a novel hCoV associated with severe respiratory diseases known as severe acute respiratory syndrome-CoV (SARS-CoV) emerged in China [12,13], most likely from bats via civet cats into humans [14], and spread globally causing the SARS epidemic causing >8000 cases and $~ 800$ deaths. Since then, there has been focused research on CoVs, and two previously unidentified hCoVs were discovered; hCoVNL63 in 2004 [15] and hCoV-HKU1 in 2005 [16], as other human respiratory pathogens that cause the common cold. In 2012, a novel zoonotic CoV known as the Middle East respiratory syndromeCoV (MERS-CoV) emerged in Saudi Arabia and [17] spread to 27 countries causing 2500 
confirmed infections and $~ 860$ deaths with large outbreaks, mainly in healthcare facilities and mostly in Saudi Arabia [18].

More recently, on January $7^{\text {th }}, 2020$, a previously unknown $\mathrm{hCoV}$, named severe acute respiratory syndrome-CoV-2 (SARS-CoV-2), was identified as a causative agent of an acute respiratory syndrome, known as Coronavirus Disease 2019 (COVID-19), in the city of Wuhan, the capital of Hubei province and one of the largest cities in China, further confirming the imminent dangers risk of zoonotic CoVs on humans [19]. Within a few weeks, the virus spread from Wuhan to different provinces in China and subsequently spread outside China reaching over 201 countries to date. The global rapid and continuous spread of SARS-CoV-2 has led the World Health Organization (WHO) to declare COVID-19 outbreak as a public health emergency of international concern (PHEIC) by the end of January 2020, and as global pandemic on March $11^{\text {th }}$, 2020. As of March $25^{\text {th }}, 2020$, SARS-CoV-2 has caused more than 550,000 confirmed cases and $\sim 25,000$ associated deaths. While the initial epicenter of the outbreak was Wuhan, multiple potential epicenters of the disease have been observed outside China since then including Italy, Spain, Iran, the USA, France, and Germany; strongly highlighting the quick spread and high transmissibility of the virus to new territories. Nonetheless, the situation in Iran and Egypt raises fears of further virus spread in the Middle East mostly due to the possible insufficient testing. Indeed, the first case of COVID-19 in Saudi Arabia was detected in a traveler returning from Iran on March $2^{\text {nd }}, 2020$. As of March $27^{\text {th }}$, the country has reported 1104 cases with three deaths (Figure 1). The majority of cases in Saudi Arabia appears to be in returning travelers and their immediate contacts so far [20].

SARS-CoV-2 belongs to betacoronaviruses, which also contains the other two highly pathogenic CoVs: SARS-CoV and MERS-CoV. It is also genetically close to SARS-related bat CoVs, 
suggesting a bat origin of the virus similar to SARS-CoV and MERS-CoV as well [21]. However, SARS-CoV-2 could have been transmitted to humans through a yet to be identified intermediate host(s). Transmission of SARS-CoV-2 in humans occurs primarily through droplets generated during coughing or sneezing or indirectly through contact with contaminated surfaces [22]. Experimentally, it was shown that the virus may remain viable for 4 and 24 hours on cardboard and copper, respectively, and for up to three days on plastic and steel surfaces; although with a remarkable decline in the virus titer [23]. It was also shown that the virus can be shed in asymptomatic patients during the incubation period, which is estimated to last up to 14 days [24]. These studies suggest high transmissibility of this novel virus which could justify its contagiousness and rapid global widespread.

\section{Saudi Arabia response to COVID-19}

The ongoing explosive spread of SARS-CoV-2 throughout the world represents a critical challenge for all countries. Like the H1N1 pandemic in 2009, SARS-CoV-2 is reaching most countries around the world. While stopping the spread of the virus is becoming extremely difficult according to the WHO, countries have to implement strict measures to mitigate its impact. In China, for example, since the SARS-CoV-2 outbreak broke out in Wuhan in late December 2019, historical strict control and preventive measures were undertaken including a mass lockdown of cities and people, shutdown of schools and businesses, travel and transportation restrictions, building hospitals in days, rapid deployments of testing protocols, and using novel technologies to trace all cases [25]. All these extreme measures and massive responses applied with rigorous and innovative approaches have resulted in a dramatic slow spread of the virus across China. Currently, 
China is reporting few, if any, new local cases of SARS-CoV-2. On the other hand, the pandemic is spreading rapidly elsewhere around the world.

Saudi Arabia was among the first countries to implement precautionary measures to prevent SARS-CoV-2 introduction into the country and to mitigate its impact once it arrives. Early before reporting the first case on March $2^{\text {nd }}, 2020$, Saudi Arabia showed an improved preparedness for the outbreak by undertaking unprecedented precautionary measures. As the host for millions of visiting Muslims and the two holiest Islamic shrines in Makkah and Madinah, Saudi Arabia is obliged to protect the health of not only its citizens and residents but also all visitors from around the world. Hajj and Umrah, which are considered an obligation for all adult Muslims worldwide, is one of the largest mass gatherings in the world. Umrah is performed by thousands of Muslims daily while Hajj is an annual pilgrimage to the holy city of Makkah that takes place around the $12^{\text {th }}$ lunar month of each year and lasts for 5 days and performed by more than 2 million Muslims annually. Although the government of Saudi Arabia undertakes enormous efforts each year to ensure the healthiness, safety and security of pilgrims, Umrah and Hajj still represent a high risk for spreading infectious diseases globally $[26,27]$. Thus, on February $6^{\text {th }}$, the first early proactive decision issued by the Saudi government is to prevent the spread of SARS-CoV-2 pandemic by stopping all direct flights between Saudi Arabia and China [28]. On February $27^{\text {th }}$, the government suspended entry of all international Umrah pilgrims and tourists and monitored all entry points to Makkah and Madinah. By February $28^{\text {th }}$, Saudi Arabia banned inbound travel of individuals from SARS-CoV-2 affected countries, including GCC citizens who have traveled to affected countries. Such decisions would not only minimize the chance of SARS-CoV-2 introduction into the country but would also prevent the exportation of cases to many unaffected countries. 
Despite these restrictions, on March $2^{\text {nd }}$, Saudi Arabia reported its first COVID-19 confirmed case in a traveler returning from Iran through Bahrain without declaring travel history to Iran. In its continuous effort to minimize the devastating effects of COVID-19 and to curb the spread of the pandemic, Umrah was completely suspended by March $4^{\text {th }}$ and the two holy mosques in Makkah and Madinah were put to daily closure for cleaning and disinfection by March $5^{\text {th }}$. On March $8^{\text {th }}$, the Saudi government shifted schools and universities from in-person classes to remote learning and virtual classrooms. This was accompanied by a travel ban to all affected countries and putting in place mandatory quarantine for passengers who already arrived from these countries. On March $9^{\text {th }}$, Saudi Arabia pledged 10 million US dollars to the WHO to help in their efforts to fight the pandemic. By March $12^{\text {th }}$, all social and governmental gatherings and events were suspended or postponed including the Saudi-African and Arab-African summits. Subsequently, all international and domestic air travels, sports events, workplaces (except security and health sectors) were suspended. In addition, the five daily prayers in all hundreds of thousands of mosques across the country were banned and all Muslims in Saudi Arabia were requested by religious authorities to pray at homes for the very first time in the history of the Kingdom. This is a remarkable step considering that Saudi Arabia law is based on the Share'a low and most Saudis do perform their prayers in masses in mosques. Digital health was quickly activated and utilized for several services such as a "my health" app that allows people to seek medical help and receive medical prescriptions without the need to visit the medical centers. It is important to note that all of these measures were taken while the number of confirmed cases in KSA was still less than 300, in a country with over 30 million population [29].

The role of social media is not to be neglected as the percentage of Twitter users in Saudi Arabia is among the highest in the world [30]. Twitter was flooded with awareness campaigns; and 
ministers, celebrities, academics, medics, and others have been tweeting "Stay Home" and "flatten the curve". Other governmental local awareness messages also are being sent every day using text messages and media outlets. Eventually, when the number of cases reached 500, on March $24^{\text {th }}$, Saudi Arabia issued a curfew from $7 \mathrm{pm}$ to 6 am and imposed a strong financial penalty on lawbreakers. In its last struct actions, Saudi Arabia issued a lockdown on major cities such Riyadh, Makkah and Madinah and banned travels between all of its 13 provinces.

So far, the virus spread in the country is considered limited as compared to other countries, with a total of 900 confirmed cases and two deaths reported as of March $25^{\text {th }}, 2020$. It is also noteworthy that the majority of cases in Saudi Arabia are returning travelers. Figure 2 summarizes the control and preventive measures taken by Saudi Arabia in response to the SARS-CoV-2 pandemic. More measures are also expected as the situation evolves. While Saudi Arabia has not announced its decision concerning the 2020 Hajj, the continuous spread of SARS-CoV-2 globally may lead the government of Saudi Arabia to suspend this year's Hajj; if it occurs, this would be the first time in recent history. Saudi King has called for an online conference for the G20 countries leaders to join a conference in supporting the global efforts against the impact of COVID-19 worldwide. In this extraordinary meeting, the leaders of the G20 countries committed to take all necessary measures and ensure adequate financing to contain the COVID-19 pandemic.

\section{MERS emergence and lesson learned}

It is apparent that the experience learned by China from dealing with several emerging infectious diseases including the emergence and spread of SARS-CoV in 2002/2003 has enabled China to better respond to the current SARS-CoV-2 pandemic [25]. Similarly, Saudi Arabia has 
experienced the outbreak of MERS-CoV which was first identified in Jeddah in 2012 and is still endemic in the country [17]. To date, MERS-CoV has caused more than 2500 cases including 860 deaths with a high mortality rate of 35\%. Although MERS-CoV has spread to 27 countries, nearly 90\% of the cases have occurred in Saudi Arabia. Over the course of seven years, Saudi Arabia has gained tremendous experience from the MERS-CoV endemic. For example, after the discovery of the MERS-CoV, the Saudi Ministry of Health $(\mathrm{MoH})$ has promptly established a command and control center and accelerated the establishment of the Saudi Center for Disease Control and Prevention (SCDC), which are now operational and are in the frontline in the country response to SARS-CoV-2. In addition, in response to MERS-CoV outbreaks in 2015, the Saudi MoH launched the National Health Laboratory (NHL). The Saudi NHL is a reference laboratory with an emphasis on providing advanced diagnostics to infectious diseases. The NHL also is expected to have the county's first high biocontainment laboratories [31].

One other impact of MERS epidemic was the improvement of biosafety in diagnostic laboratories and the application of strict infection prevention and control (IPC) systems in all hospitals across the country. Some may comment that "MERS-CoV has a blessing of improving IPC in Saudi hospitals" Additionally, the Saudi MoH has designated regional hospitals for the isolation and treatment of MERS patients. These hospitals are now well prepared and started to receive COVID19 patients.

Furthermore, the medical and scientific research community demonstrated marvelous efforts in the understanding and control of MERS-CoV in the past few years, as evident by more than 480 publications to date [32]. A good number of these articles were published in high-quality medical and scientific journals and covered both translational and clinical research. Saudi Arabia has started its first ever phase I clinical trial on a vaccine candidate for MERS-CoV (Clinical trial 
NCT04170829); The MIRACLE trial has started and been ongoing to evaluate antiviral therapeutics in severely infected MERS patients (Clinical trial NCT02845843) and now will include COVID-19 patients, and two vaccines have been developed and tested in animal studies $[33,34]$; another vaccine has been tested in camels as a one-health veterinary vaccine, since camels are the intermediate host for MERS-CoV [35], in addition to several epidemiologic and observational studies.

This advancement in research was coupled and supported by improved regulations at the Saudi FDA, MoH, and other regulators. Saudi FDA has supported the approval of the first vaccine Phase I clinical trial in the history of the country; despite being a very young organization by itself. MoH has advocated for national IRB approval for MERS research trials. King Abdulaziz City for Science and Technology (KACST) has enabled the advancement of R\&D by building a national center for vaccine development and manufacturing and further supported the newly established BADIR Program in supporting the acceleration and incubation of life science discoveries [36].

As in many countries, many areas of improvement are still ahead. More support and encouragement of fast and effective research and development is needed to enable the country to be more prepared in the case of epidemics and pandemics. Except for one biosafety level 3 (BSL3) laboratory in an academic institution, high containment laboratories such as BSL-3/4 and animal BSL-3 (ABSL-3) are still lacking in the country. More advanced industrial-scale development grants are needed with networking between academia and industry.

\section{Conclusion and Remarks}

Saudi Arabia and China are the two countries that have witnessed the documented emergence of novel highly pathogenic coronavirus epidemics in recent years. These countries must have 
accumulated a frontline experience in dealing with and preparing for coronaviruses epidemics. MERS-CoV emergence in 2012 with continuing but controlled epidemic has put Saudi Arabia on a high sense of alert and readiness to take whatever measures to curb COVID-19 spread. Saudi Arabia has imposed a number of extreme measures on social movement, social gathering, religious gathering, travel, and businesses way before the first COVID-19 case in the country or before the number of cases reached 100 cases. It is expected that Saudi Arabia would have a flatter curve than many of other countries and may be able to control the pandemic quicker than others. MERS epidemics helped Saudi Arabia to have better alerted public health and infection control policies and measures as well as educating the general public on outbreaks and viral epidemics. Saudi Arabia has improved in terms of clinical and scientific research on epidemics, but it has yet a long way to go in building its appropriate biocontainment laboratories and moving into better governance of research and development. 


\section{References}

[1] Fehr AR, Perlman S. Coronaviruses: An overview of their replication and pathogenesis. Coronaviruses Methods Protoc., 2015, p. 1-23. doi:10.1007/978-1-4939-2438-7_1.

[2] de Groot RJ, Baker SC, Baric RS, Brown CS, Drosten C, Enjuanes L, et al. Middle East Respiratory Syndrome Coronavirus (MERS-CoV): Announcement of the Coronavirus Study Group. J Virol 2013. doi:10.1128/JVI.01244-13.

[3] Hudson CB, Beaudette FR. Infection of the cloaca with the virus of infectious bronchitis. Science (80- ) 1932. doi:10.1126/science.76.1958.34-a.

[4] Pensaert MB, de Bouck P. A new coronavirus-like particle associated with diarrhea in swine. Arch Virol 1978. doi:10.1007/BF01317606.

[5] Saif LJ. Mucosal immunity: An overview and studies of enteric and respiratory coronavirus infections in a swine model of enteric disease. Vet. Immunol. Immunopathol., 1996. doi:10.1016/S0165-2427(96)05702-9.

[6] Cavanagh D. Coronaviruses in poultry and other birds. Avian Pathol 2005. doi:10.1080/03079450500367682.

[7] Liu L, Hägglund S, Hakhverdyan M, Alenius S, Larsen LE, Belák S. Molecular epidemiology of bovine coronavirus on the basis of comparative analyses of the $\mathrm{S}$ gene. $\mathrm{J}$ Clin Microbiol 2006. doi:10.1128/JCM.44.3.957-960.2006.

[8] Perlman S, Netland J. Coronaviruses post-SARS: Update on replication and pathogenesis. Nat Rev Microbiol 2009. doi:10.1038/nrmicro2147.

[9] Cui J, Li F, Shi ZL. Origin and evolution of pathogenic coronaviruses. Nat Rev Microbiol 2019. doi:10.1038/s41579-018-0118-9.

[10] McIntosh K, Dees JH, Becker WB, Kapikian AZ, Chanock RM. Recovery in tracheal organ cultures of novel viruses from patients with respiratory disease. Proc Natl Acad Sci U S A 1967. doi:10.1073/pnas.57.4.933.

[11] Hamre D, Procknow JJ. A New Virus Isolated from the Human Respiratory Tract. Proc Soc Exp Biol Med 1966. doi:10.3181/00379727-121-30734.

[12] Peiris JSM, Lai ST, Poon LLM, Guan Y, Yam LYC, Lim W, et al. Coronavirus as a possible cause of severe acute respiratory syndrome. Lancet 2003. doi:10.1016/S01406736(03)13077-2.

[13] Drosten C, Günther S, Preiser W, Van der Werf S, Brodt HR, Becker S, et al. Identification of a novel coronavirus in patients with severe acute respiratory syndrome. $\mathrm{N}$ Engl J Med 2003. doi:10.1056/NEJMoa030747.

[14] Li W, Shi Z, Yu M, Ren W, Smith C, Epstein JH, et al. Bats are natural reservoirs of SARS-like coronaviruses. Science (80- ) 2005. doi:10.1126/science.1118391.

[15] Van Der Hoek L, Pyrc K, Jebbink MF, Vermeulen-Oost W, Berkhout RJM, Wolthers KC, et al. Identification of a new human coronavirus. Nat Med 2004. doi:10.1038/nm1024. 
[16] Woo PCY, Lau SKP, Chu C -m., Chan K -h., Tsoi H -w., Huang Y, et al. Characterization and Complete Genome Sequence of a Novel Coronavirus, Coronavirus HKU1, from Patients with Pneumonia. J Virol 2005. doi:10.1128/jvi.79.2.884-895.2005.

[17] Zaki AM, Van Boheemen S, Bestebroer TM, Osterhaus ADME, Fouchier RAM. Isolation of a novel coronavirus from a man with pneumonia in Saudi Arabia. N Engl J Med 2012. doi:10.1056/NEJMoa1211721.

[18] [accessed March 25 2020]. The world health organization (WHO). Middle East respiratory syndrome coronavirus (MERS-CoV) MERS Monthly Summary n.d. https://www.who.int/emergencies/mers-cov/en/ (accessed March 25, 2020).

[19] Zhu N, Zhang D, Wang W, Li X, Yang B, Song J, et al. A novel coronavirus from patients with pneumonia in China, 2019. N Engl J Med 2020. doi:10.1056/NEJMoa2001017.

[20] Saudi center for disease prevention and control (SCDC) n.d. https://covid19.cdc.gov.sa/daily-updates/ (accessed March 25, 2020).

[21] Zhou P, Yang X-L, Wang X-G, Hu B, Zhang L, Zhang W, et al. A pneumonia outbreak associated with a new coronavirus of probable bat origin. Nature 2020. doi:10.1038/s41586-020-2012-7.

[22] Xu Y. Unveiling the Origin and Transmission of 2019-nCoV. Trends Microbiol 2020. doi:10.1016/j.tim.2020.02.001.

[23] van Doremalen N, Bushmaker T, Morris DH, Holbrook MG, Gamble A, Williamson BN, et al. Aerosol and Surface Stability of SARS-CoV-2 as Compared with SARS-CoV-1. N Engl J Med 2020. doi:10.1056/NEJMc2004973.

[24] Bai Y, Yao L, Wei T, Tian F, Jin DY, Chen L, et al. Presumed Asymptomatic Carrier Transmission of COVID-19. JAMA - J Am Med Assoc 2020. doi:10.1001/jama.2020.2565.

[25] Xiao Y, Torok ME. Taking the right measures to control COVID-19. Lancet Infect Dis 2020. doi:10.1016/S1473-3099(20)30152-3.

[26] Memish ZA, Zumla A, Alhakeem RF, Assiri A, Turkestani A, Al Harby KD, et al. Hajj: Infectious disease surveillance and control. Lancet 2014. doi:10.1016/S01406736(14)60381-0.

[27] Hashem AM, Al-Subhi TL, Badroon NA, Hassan AM, Bajrai LHM, Banassir TM, et al. MERS-CoV, influenza and other respiratory viruses among symptomatic pilgrims during 2014 Hajj season. J Med Virol 2019. doi:10.1002/jmv.25424.

[28] Saudi Arabia bars travel to China amid coronavirus n.d. https://www.arabnews.com/node/1623851/saudi-arabia (accessed March 25, 2020).

[29] World Population Review. Saudi Arabia Population 2018 (Demographics, Maps, Graphs). World Popul Rev 2018.

[30] Leading countries based on number of Twitter users as of January 2020 n.d.

[31] Minitry of Health, National Health Laboratory n.d. 
https://www.moh.gov.sa/en/Ministry/MediaCenter/News/Pages/News-2015-03-25002.aspx (accessed March 25, 2020).

[32] Fluctuating funding and flagging interest hurt coronavirus research, leaving crucial knowledge gaps n.d. https://www.statnews.com/2020/02/10/fluctuating-funding-andflagging-interest-hurt-coronavirus-research/د (accessed March 25, 2020).

[33] Al-Amri SS, Abbas AT, Siddiq LA, Alghamdi A, Sanki MA, Al-Muhanna MK, et al. Immunogenicity of Candidate MERS-CoV DNA Vaccines Based on the Spike Protein. Sci Rep 2017. doi:10.1038/srep44875.

[34] Hashem AM, Algaissi A, Agrawal AS, Al-Amri SS, Alhabbab RY, Sohrab SS, et al. A Highly Immunogenic, Protective, and Safe Adenovirus-Based Vaccine Expressing Middle East Respiratory Syndrome Coronavirus S1-CD40L Fusion Protein in a Transgenic Human Dipeptidyl Peptidase 4 Mouse Model. J Infect Dis 2019. doi:10.1093/infdis/jiz137.

[35] Alharbi NK, Qasim I, Almasoud A, Aljami HA, Alenazi MW, Alhafufi A, et al. Humoral Immunogenicity and Efficacy of a Single Dose of ChAdOx1 MERS Vaccine Candidate in Dromedary Camels. Sci Rep 2019. doi:10.1038/s41598-019-52730-4.

[36] Groundbreaking for first R\&D vaccine center in the Middle East takes place at KAUST. https://innovation.kaust.edu.sa/groundbreaking-for-first-r-d-vaccine-center-in-the-middleeast-takes-place-at-kaust/ (accessed March 25, 2020). 


\section{Figure Legends:}

Figure 1: Total COVID-19 confirmed cases in Saudi Arabia and other major countries as of March 27 $7^{\text {th }}, 2020$. Data were collected from the World Health Organization and ministries of health in these countries.

Figure 2: Daily confirmed COVID-19 cases in Saudi Arabia and the major preventive and control measures taken by the government and health authorities of Saudi Arabian as of March $27^{\text {th }}, 2020$. Data are from the Ministry of Health, Saudi Center of Disease control and prevention, and Saudi Press Agency. 


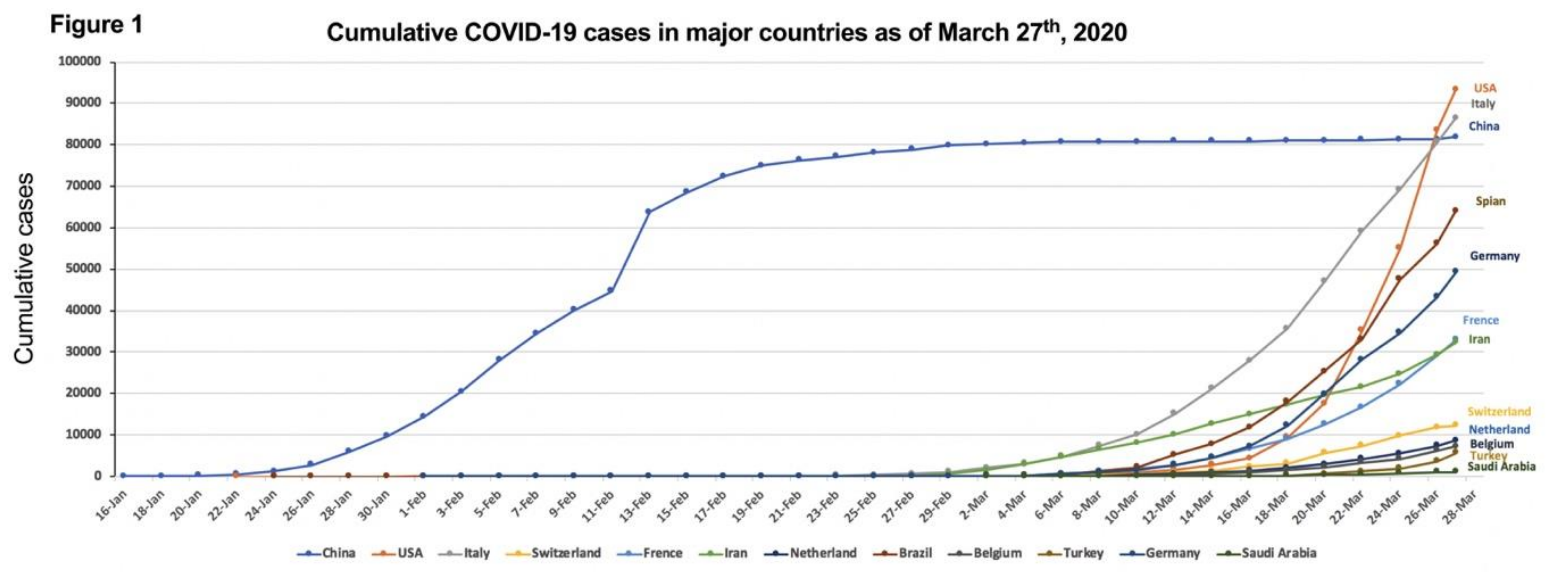

Figure 2

Daily confirmed cases and major preventive

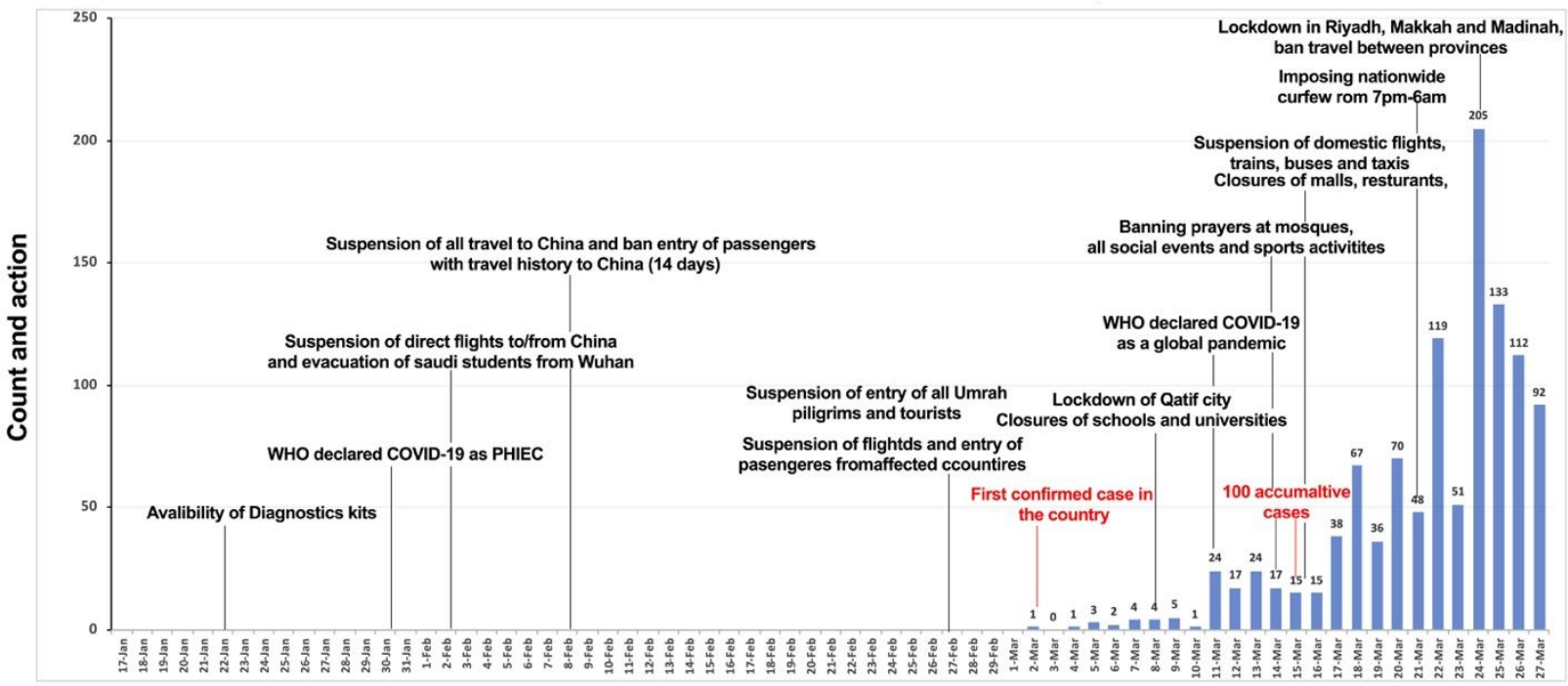

